\title{
Classical double copy of worldline quantum field theory
}

\author{
Canxin Shi $\odot^{*}$ and Jan Plefka $\odot^{\dagger}$ \\ Institut für Physik und IRIS Adlershof, Humboldt-Universität zu Berlin, \\ Zum Großen Windkanal 2, 12489 Berlin, Germany
}

(Received 7 October 2021; accepted 19 November 2021; published 7 January 2022)

\begin{abstract}
The recently developed worldline quantum field theory (WQFT) formalism for the classical gravitational scattering of massive bodies is extended to massive, charged point particles coupling to biadjoint scalar field theory, Yang-Mills theory, and dilaton-gravity theory. We establish a classical double copy relation in these WQFTs for classical observables (deflection, radiation). The biadjoint scalar field theory fixes the locality structure of the double copy from Yang-Mills to dilaton gravity. Using this, the eikonal scattering phase (or free energy of the WQFT) is computed to next-to-leading order (NLO) in coupling constants using the double copy as well as directly finding full agreement. We clarify the relation of our approach to previous studies in the effective field theory formalism. Finally, the equivalence of the WQFT double copy to the double copy relation of the classical limit of quantum scattering amplitudes is shown explicitly up to NLO.
\end{abstract}

DOI: 10.1103/PhysRevD.105.026007

\section{INTRODUCTION}

Finding a unified field theory of gauge bosons and gravitons has been a holy grail of physics since the days of Einstein, Heisenberg, and Pauli. While Yang-Mills theory and Einstein gravity share common features such as local symmetries, their actions appear to be very different. In particular, their perturbative quantization in weakly interacting situations strongly differ; while the Feynman diagrammatic expansion for gravity is notoriously involved and not renormalizable, the Yang-Mills case is under good control and the basis of high precision predictions for scattering experiments at ever increasing orders in perturbation theory. This is why the construction of Bern-Carrasco-Johansson (BCJ) $[1,2]$ for the integrand of quantum gravitational scattering amplitudes in terms of a double copy of Yang-Mills ones has been highly inspirational and points to a surprisingly direct and intimate connection between these two fundamental theories of nature, see [3] for a recent review. Concretely, the double copy construction arranges the building blocks of gluon scattering amplitudes in terms of kinematic numerators, color factors, and scalar propagators in such a fashion, that the kinematic numerators obey identities akin to the Jacobi

\footnotetext{
*canxin.shi@physik.hu-berlin.de

jan.plefka@hu-berlin.de
}

Published by the American Physical Society under the terms of the Creative Commons Attribution 4.0 International license. Further distribution of this work must maintain attribution to the author(s) and the published article's title, journal citation, and DOI. Funded by SCOAP . identity constitutional for the color factors resulting from the color gauge symmetry. Replacing the color factors by the thereby identified kinematic numerators of the gluon amplitudes then yields the integrands of scattering amplitudes in axion-dilaton gravity (or $\mathcal{N}=0$ supergravity, i.e., the low energy limit of the bosonic string). Formal proofs of the double copy have been provided at tree level using a variety of methods [4]. This "color-kinematic duality" has been extended to a large class of theories, and the question of which (gravitational) theories admit a double copy is an interesting and, in general, open one. At the same time, the double copy presents a very efficient tool to construct amplitude integrands in (super)gravity to very high loop orders, see [3] for an account. Yet, its deeper nature, in particular, the nature of the "kinematic algebra", remains ill understood and is a subject of intense research [5] in the modern amplitude program, see [6] for reviews.

The double copy relation for quantum scattering amplitudes leads to a natural challenge for classical general relativity. Namely, is there a classical double copy transforming solutions of Yang-Mills (or Maxwell's) theory to gravity (with a dilaton) as well? In fact a number of such constructions has been provided [7], most prominently perhaps for the Kerr-Newman solution of a spinning black hole [8]. Yet, from the quantum origin of the colorkinematic duality, it should be clear that the perturbative nature of the classical solution, i.e., the systematic expansion about a flat Minkowski background (known as the post-Minkowskian expansion in general relativity), should be central for the existence of a classical double copy prescription. Fascinatingly, this is also the domain relevant for analytic gravitational wave physics, describing the 
inspiral (or scattering) of two massive bodies (black holes, neutron stars, or stars). The emitted gravitational radiation in that two-body process is detectable in present and future gravitational wave detectors (without the dilaton, and for the bound system in the post-Newtonian expansion, which is a combined weak field and slow motion expansion).

The approach of constructing perturbative classical solutions via the double copy was pioneered in [9]. The scattering of two point particles via dilaton and graviton interactions was constructed via a double copy of the perturbative solutions of the Yang-Mills equations coupled to point particles carrying color charge. This was further extended and clarified to the next-to-next-to leading order (NNLO) by Shen in [10]. Spin effects and extensions were studied in [11-13], and the analogue problem in biadjoint scalar field theory was considered in [14,15]. All these approaches operate at the level of the equations of motion; i.e., one perturbatively solves both the field and particle equations of motion. An alternative route was taken in the works [16,17], involving the present authors, where a path integral based approach was taken. Here, starting from the actions describing the coupling of massive, charged particles to Yang-Mills or dilaton gravity, the force mediating fields (gluons, dilatons, and graviton) were integrated out yielding an effective action for the point particles, thereby taking the classical $\hbar \rightarrow 0$ limit. It was shown at LO and NLO that the resulting effective action could be obtained by a suitably generalized double copy prescription [16] taking inspiration from the amplitudes approach. Concretely, the need for a trivalent graph structure was artificially introduced via delta functions on the worldline for higher valence worldline-bulk field vertices. Yet, this double copy prescription was shown to break down for the effective action at the NNLO [17]. It was speculated in [17] that the reason for this breakdown lies in the attempt of double copying a gauge variant and off-shell quantity-the effective action-which is at tension with the on-shell nature of the scattering amplitude double copy.

Returning to the realm of applying quantum field theory based techniques to the post-Minkowskian perturbative gravitational scattering problem, an approach termed worldline quantum field theory (WQFT) was put forward recently [18] that explains the relation between the two presently common approaches employing the classical limit of scattering amplitudes, see, e.g., [19-22], and the PM effective field theory (EFT) approach, see, e.g., [23]. In essence it starts out from a first quantized description of the matter field's (scalar, spinor, vector) propagator in a gravitational background à la Feynman-Schwinger and demonstrates how this leads in a classical limit to the effective field theory description. Yet, the WQFT formalism not only is of conceptual relevance (introducing an approximate supersymmetry in the description of spin for black holes and neutron stars [24]), but it also provides a very efficient tool to quickly arrive at the classical observables of the scattering process without the need to go through an iterative solution of the equations of motion in the standard EFT approach or to deal with the subtleties of the classical limit in the amplitudes based approach. As such, the deflection, the spin kick, or the explicit gravitational Bremsstrahlung waveform as well as the eikonal being the generating function of these have been established at NLO [24-26].

In this work, we therefore address the conceptually important question whether a double copy prescription exists for the WQFT as well? On the face of it, this is to be expected, as the WQFT may be thought of as a (partially) first order form of the scattering amplitude problem. For this, we first settle for the relevant worldline quantum field theories in Sec. II. This includes, in particular, the case of the worldline coupled biadjoint scalar field theory which we indeed require in order to separate the kinematic numerators from the propagator terms in the Yang-Mills case - a prerequisite for the double copy construction. In Sec. II, we establish the relevant Feynman rules for all three WQFTs, coupling to biadjoint scalars, Yang-Mills and dilaton gravity as well as point out the relevance of the eikonal or free energy of the WQFT as a generating function for the observable of the particle's deflections. In Sec. III, we develop the classical double copy prescription for WQFTs and construct the eikonal at the NLO level. Section IV is devoted to the lift of our results for the (threeparticle) eikonal to the radiative waveform, and in Sec. V, we detail the relation of our WQFT double copy to the established one for the quantum scattering amplitudes. After concluding, we collect our conventions in the Appendix.

\section{WORLDLINE QUANTUM FIELD THEORIES}

We apply the worldline quantum field theory formalism [18] to massive point particles coupled to biadjoint scalar field theory (BS), Yang-Mills theory (YM), and dilatongravity theory (DG). Compactly, the actions for the three theories may be written as

$$
S^{\mathrm{WQFT}}=S^{\mathrm{BS} / \mathrm{YM} / \mathrm{DG}}+\sum_{i} S_{i}^{\mathrm{cc} / \mathrm{pc} / \mathrm{pm}},
$$

where $S^{\mathrm{BS} / \mathrm{YM} / \mathrm{DG}}$ is the respective field theory action and $S_{i}^{\mathrm{cc} / \mathrm{pc} / \mathrm{pm}}$ the respective $i$ th particle worldline action. Note that multiple worldlines are included in Eq. (1) in order to allow for interactions. We now focus on the worldline actions $S_{i}^{\mathrm{cc} / \mathrm{pc} / \mathrm{pm}}$ and delegate the rather well known field theory actions to Appendix A.

The action of a massive point charge coupled to a nonAbelian gauge field $A_{\mu}^{a}$ is $[27,28]$

$S^{\mathrm{pc}}=-\int \mathrm{d} \tau\left(\frac{m}{2}\left(e^{-1} \dot{x}^{2}+e\right)-i \Psi \dot{\Psi}-g \dot{x}^{\mu} A_{\mu}^{a} C^{a}\right)$, 
where $e(\tau)$ is the einbein, and the dot over a symbol denotes a derivative with respect to $\tau$. The "color wave function" $\Psi_{\alpha}(\tau)$ is an auxiliary field carrying the color degrees of freedom of the particle, the $\alpha, \beta, \ldots=1, \ldots, d_{R}$ are indices of the $d_{R}$ dimensional representation of the gauge group, and $C^{a}(\tau)=\Psi^{\dagger \alpha}\left(T^{a}\right)_{\alpha} \beta \Psi_{\beta}$ is the associated color charge that determines the coupling to the gauge field $A_{\mu}^{a}(x)$. We take the generators $\left(T^{a}\right)_{\alpha}{ }^{\beta}$ to be in the fundamental of $S U(N)$ such that $d_{R}=N$, and the adjoint indices $a, b, \ldots=1, \ldots N^{2}-1{ }^{1}$ This action is invariant under the reparametrization of $\tau$. The kinetic term can be transformed into the more familiar form $-m \int \mathrm{d} \tau \sqrt{\dot{x}^{2}}$ by solving the algebraic equations of motion for the einbein $e(\tau)$ and reinserting the solution into the action. However, for convenience we fix $e(\tau)=1$ such that $\dot{x}^{2}=1$, and $\tau$ is then the proper time.

Similarly, the action of a worldline minimally coupled to dilaton gravity reads

$$
S^{\mathrm{pm}}=-\frac{m}{2} \int \mathrm{d} \tau\left(e^{-1} e^{2 \kappa \varphi} g_{\mu \nu} \dot{x}^{\mu} \dot{x}^{\nu}+e\right),
$$

where $\varphi(x)$ is the dilaton, and $e(\tau)$ is again the einbein. The coupling constant is $\kappa=\sqrt{32 \pi G}$, where $G$ is Newton's constant. Again, upon integrating out $e(\tau)$, we arrive at the more common form of the action $-m \int \mathrm{d} \tau e^{\kappa \varphi} \sqrt{g_{\mu \nu} \dot{x}^{\mu} \dot{x}^{\nu}}$. We gauge fix $e(\tau)=1$ so that $e^{2 \kappa \varphi} g_{\mu \nu} \dot{x}^{\mu} \dot{x}^{\nu}=1$. In the weak gravitational field limit, we expand

$$
g_{\mu \nu}=\eta_{\mu \nu}+\kappa h_{\mu \nu}
$$

where $\eta_{\mu \nu}$ is the flat-space Minkowskian metric. Because the dilaton appears as an exponent in the action, the interaction terms become cumbersome in perturbation theory of both the worldline and field theory actions. To simplify the calculation, we adopt the gravity gauge-fixing term and field redefinitions of $\left\{\varphi, h_{\mu \nu}\right\}$ introduced in [16] Eqs. (51)-(56). This will decouple the worldline from $\varphi$ up to quadratic order, as well as recast the cubic selfinteraction of $h_{\mu \nu}$ into a simpler form. The redefined field theory action can be found in (A11) of Appendix A. The worldline action in terms of the redefined fields then reads

$S^{\mathrm{pm}}=-\frac{m}{2} \int \mathrm{d} \tau\left(\dot{x}^{2}+\kappa h_{\mu \nu} \dot{x}^{\mu} \dot{x}^{\nu}+\frac{\kappa^{2}}{2} h_{\mu \rho} h_{\nu}{ }^{\rho} \dot{x}^{\mu} \dot{x}^{\nu}\right)+\mathcal{O}\left(\kappa^{3}\right)$.

The indices are lowered or raised by the Minkowskian metric.

\footnotetext{
${ }^{1}$ In fact, specifying the gauge group and the representation for the matter are not necessary. We can, in principal, pick any group and representation.
}

Finally, let us introduce the massive point particle coupling to a biadjoint scalar field theory. Here, we use the biadjoint scalar theory to identify the double copy kernel as introduced in [10]. A point particle interacting with a biadjoint scalar field (WBS) is described by $[10,15]$

$$
\begin{aligned}
S^{\mathrm{cc}}= & -\int \mathrm{d} \tau\left(\frac{m}{2}\left(e^{-1} \dot{x}^{2}+e\right)-i \Psi \dot{\Psi}\right. \\
& \left.-i \tilde{\Psi} \dot{\tilde{\Psi}}-e \frac{y}{m} \phi_{a \tilde{a}} C^{a} \tilde{C}^{\tilde{a}}\right),
\end{aligned}
$$

where $y$ is the coupling constant, and $\phi_{a \tilde{a}}(x)$ is the biadjoint scalar field carrying two distinct color indices $a$ and $\tilde{a}$ related to the color and dual-color gauge groups, respectively. $\Psi_{\alpha}(\tau)$ and $\tilde{\Psi}_{\tilde{\alpha}}(\tau)$ are the color and dual-color wave functions. The corresponding charges are defined in a similar way as in $S^{\mathrm{pc}}$ of (2): $C^{a}=\Psi^{\dagger} T^{a} \Psi$ and $\tilde{C}^{\tilde{a}}=\tilde{\Psi}^{\dagger} \tilde{T}^{\tilde{a}} \tilde{\Psi}$. Note that setting $e(\tau)=1$ in this case will enforce the constraint $\dot{x}^{2}+\frac{2 y}{m^{2}} \phi_{a \tilde{a}} C^{a} \tilde{C}^{\tilde{a}}=1$.

In the worldline quantum field theory (WQFT) formalism, describing the scattering of two particles, we expand the coordinate $x^{\mu}(\tau)$ along a straight line trajectory background

$$
x^{\mu}(\tau)=b^{\mu}+v^{\mu} \tau+z^{\mu}(\tau),
$$

with $v^{2}=1$ and the fluctuation $z^{\mu}$. Note that the straight line background solves the equations of motion in the field free scenario(s) $\phi_{a \tilde{a}}=A_{\mu}^{a}=h_{\mu \nu}=\varphi=0$. We take $b \cdot v=0$ which may always be achieved upon shifting $\tau$. As explained in [18], the physical meanings of $b^{\mu}$ and $v^{\mu}$ depend on the type of the worldline propagator (advanced/ retarded/time symmetric). As in this work, our main concern for the double copy construction is the integrand; the $i \epsilon$ description of the propagators is of no direct concern. Likewise, we decompose the color wave function in the background

$$
\Psi(\tau)=\psi+\Psi(\tau)
$$

where $\psi=\Psi(-\infty)=$ const is the initial condition, and $\Psi(\tau)$ is the fluctuation that will be quantized. Consequently, the color charge is

$$
C^{a}=c^{a}+\psi^{\dagger} T^{a} \Psi+\Psi^{\dagger} T^{a} \psi+\Psi^{\dagger} T^{a} \Psi,
$$

where we have defined the background color charge $c^{a}=\psi^{\dagger} T^{a} \psi$. A similar decomposition applies to the dualcolor wave function $\tilde{\Psi}(\tau)$, and all respective dual quantities are denoted with a tilde.

In WQFT, the physical observables are computed as the expectation values of the corresponding operators. We will integrate out the BS/YM/DG fields $\phi_{a \tilde{a}} ; A_{\mu}^{a} ; h_{\mu \nu}, \varphi$ as well as all fluctuations of worldline degrees of freedom 
$z(\tau), \Psi(\tau), \tilde{\Psi}(\tau)$ in the path integral, so the results only depend on the background fields $b, v, \psi$. In the path integral, the expectation value of an operator $\mathcal{O}$ is expressed as

$$
\langle\mathcal{O}\rangle=\frac{1}{\mathcal{Z}_{\mathrm{WQFT}}} \int D[\Phi] \prod_{i} D\left[z_{i}\right]\left(D\left[\Psi_{i}, \tilde{\Psi}_{i}\right]\right) \mathcal{O} e^{i S^{\mathrm{WQFT}}},
$$

where $\Phi \in\left\{\phi_{a \tilde{a}}, A_{\mu}^{a}, h_{\mu \nu}, \varphi\right\}$ denotes the bosonic fields in the respective theories. $\mathcal{Z}_{\mathrm{WQFT}}$ is the partition function,

$$
\mathcal{Z}_{\mathrm{WQFT}}=\int D[\Phi] \prod_{i} D\left[z_{i}\right]\left(D\left[\Psi_{i}, \tilde{\Psi}_{i}\right]\right) e^{i S^{\mathrm{WQFT}}}
$$

In the binary case $(i=1,2), \mathcal{Z}_{\mathrm{WQFT}}$ may be identified with the exponentiated eikonal phase $\chi$. The momentum deflection of a particle $\Delta p_{i}^{\mu}$ can be calculated by taking the derivative of the eikonal with respect to $b_{i}^{\mu}$. Here, we claim that this relation holds for an arbitrary number of worldlines, see Appendix B. In Sec. III, we perform a double copy for the eikonal to next-to-leading order.

Since we mostly work in momentum space, it is useful to express the worldline fluctuations as

$$
\begin{aligned}
z^{\mu}(\tau) & =\int_{\omega} e^{-i \omega \tau} z^{\mu}(\omega), \\
\Psi(\tau) & =\int_{\omega} e^{-i \omega \tau} \Psi(\omega), \\
\Psi^{\dagger}(\tau) & =\int_{\omega} e^{-i \omega \tau} \Psi^{\dagger}(-\omega) .
\end{aligned}
$$

The dual-color wave function $\tilde{\Psi}$ in momentum space is defined in the same way as $\Psi$. For convenience, we use the integral shorthands $\int_{\omega}:=\int \frac{\mathrm{d} \omega}{2 \pi}, \int_{k}:=\int \frac{\mathrm{d}^{4} k}{(2 \pi)^{4}}$ as well as $\delta(\omega):=2 \pi \delta(\omega)$ and $\delta^{(4)}\left(k^{\mu}\right):=(2 \pi)^{4} \delta^{(4)}\left(k^{\mu}\right)$. When evaluated on the worldline, the generic field $\Phi$ may be expanded as

$$
\begin{aligned}
\Phi(x(\tau)) & =\int_{k} e^{i k \cdot(b+v \tau+z(\tau))} \Phi(-k)=\sum_{n=0}^{\infty} \frac{i^{n}}{n !} \int_{k} e^{i k \cdot(b+v \tau)}(k \cdot z(\tau))^{n} \Phi(-k) \\
& =\int_{k} e^{i k \cdot b} \Phi(-k)\left(e^{i k \cdot v \tau}+i \int_{\omega} e^{i(k \cdot v+\omega) \tau} k \cdot z(-\omega)\right)+\mathcal{O}\left(z^{2}\right) .
\end{aligned}
$$

We take the expansion only to linear order in $z^{\mu}$ since this is the highest term we need in this paper. A complete expression of $h_{\mu \nu}$ to all orders in $z$ may be found in [18].

Next, we extract the Feynman rules from the worldline actions. The worldline propagators are the same in all three theories,

$$
z^{\mu} \stackrel{\omega}{\longrightarrow} z^{\nu}=-\frac{i}{m} \frac{\eta^{\mu \nu}}{\omega^{2}}
$$

$$
\Psi^{\dagger} \stackrel{\omega}{\longrightarrow} \cdot \Psi=\frac{i}{\omega}
$$

The propagator of the dual field $\tilde{\Psi}$ is identical to the one for $\Psi$.

Let us now begin with the analysis of the Yang-Mills coupled WQFT. With (12) and (13), we can expand the interaction term of $S^{\mathrm{pc}}$ from Eq. (2) as

$$
\begin{aligned}
S_{\mathrm{int}}^{\mathrm{pc}}= & g \int \mathrm{d} \tau \dot{x}^{\mu}(\tau) \cdot A^{a}(x(\tau)) C^{a}(\tau) \\
= & g \int_{k} e^{i k \cdot b} v \cdot A^{a}(-k) \delta(k \cdot v) c^{a}+g \int_{k, \omega} e^{i k \cdot b} A_{\mu}^{a}(-k) \delta(k \cdot v+\omega) \\
& \times\left[i\left(\omega z^{\mu}(-\omega)+v^{\mu} k \cdot z(-\omega)\right) c^{a}+v^{\mu}\left(\psi^{\dagger} T^{a} \Psi(-\omega)+\Psi^{\dagger}(\omega) T^{a} \psi\right)\right]+\mathcal{O}\left((z, \Psi)^{2}\right),
\end{aligned}
$$

where we keep the interaction to linear order in worldline fluctuations. The Feynman rules of the worldline-gluon vertices can be directly read off from (16); below we represent the background worldline configurations $\left(b^{\mu}, v^{\mu}, c^{a}\right)$ as dashed lines.

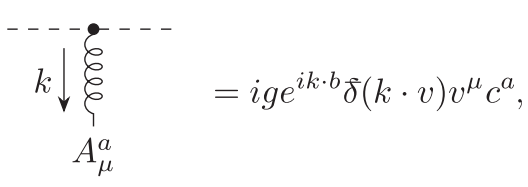




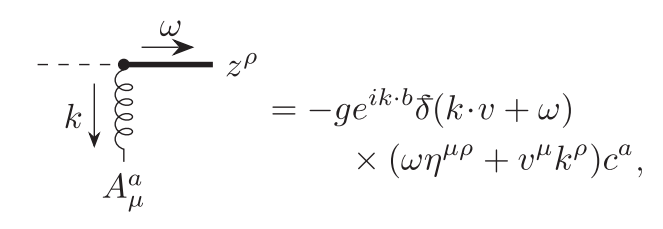

vi $\sum_{A_{\mu}^{a}}^{\stackrel{\omega}{\longrightarrow}} \Psi^{\dagger}=i g e^{i k \cdot b} \delta(k \cdot v+\omega) v^{\mu}\left(T^{a} \psi\right)$,

$$
\Psi \stackrel{\omega}{\rightarrow}{ }_{\xi_{\mu}^{\xi} \downarrow k} \downarrow^{a}=i g e^{i k \cdot b} \delta(k \cdot v-\omega) v^{\mu}\left(\psi^{\dagger} T^{a}\right) .
$$

Turning to the biadjoint scalar coupled WQFT, we can expand the worldline-scalar coupling of (6) in the same way,

$$
\begin{aligned}
S_{\mathrm{int}}^{\mathrm{cc}}= & \frac{y}{m} \int \mathrm{d} \tau \phi^{a \tilde{a}}(x(\tau)) C^{a}(\tau) C^{\tilde{a}}(\tau) \\
= & \frac{y}{m} \int_{k} e^{i k \cdot b} \phi^{a \tilde{a}}(-k) \delta(k \cdot v) c^{a} c^{\tilde{a}} \\
& +\frac{y}{m} \int_{k, \omega} e^{i k \cdot b} \phi^{a \tilde{a}}(-k) \delta(k \cdot v+\omega)\left[i k \cdot z(-\omega) c^{a} c^{\tilde{a}}\right. \\
& +\left(\psi^{\dagger} T^{a} \Psi(-\omega)+\Psi^{\dagger}(\omega) T^{a} \psi\right) c^{\tilde{a}} \\
& \left.+c^{a}\left(\tilde{\psi}^{\dagger} \tilde{T}^{\tilde{a}} \tilde{\Psi}(-\omega)+\tilde{\Psi}^{\dagger}(\omega) \tilde{T}^{\tilde{a}} \tilde{\psi}\right)\right]+\mathcal{O}\left((z, \Psi)^{2}\right) .
\end{aligned}
$$

Again, we keep only the terms that we need in this work. From the interaction (21), we extract the Feynman rules

$$
\begin{aligned}
& k \downarrow \sum_{\phi^{a b}}=\frac{i y}{m} e^{i k \cdot b} \delta(k \cdot v) c^{a} c^{\tilde{a}}, \\
& k \downarrow \sum_{\phi^{a b}}^{\stackrel{\omega}{\longrightarrow}} z^{\rho}=-\frac{y}{m} e^{i k \cdot b} \delta(k \cdot v+\omega) k^{\rho} c^{a} c^{\tilde{a}}, \\
& k \downarrow \sum_{\phi^{a b}}^{\stackrel{\omega}{\longrightarrow}} \Psi^{\dagger}=\frac{i y}{m} e^{i k \cdot b} \delta(k \cdot v+\omega)\left(T^{a} \psi\right) c^{\tilde{a}}
\end{aligned}
$$

$$
\Psi \stackrel{\omega}{\rightarrow}\left\{\begin{array}{l}
\phi^{a b} \\
\downarrow
\end{array} \mid--\frac{i y}{m} e^{i k \cdot b} \delta(k \cdot v-\omega)\left(\psi^{\dagger} T^{a}\right) c^{\tilde{a}} .\right.
$$

For vertices that involves the dual wave function, we simply use (24) or (25) and change $\Psi$ to $\tilde{\Psi}$.

In the dilaton-gravity coupled WQFT, the interaction term is remarkably simplified by the field redefinitions of $\left\{\varphi, h_{\mu \nu}\right\}$. In the end, the linear order in $h_{\mu \nu}$ is no different than the interaction term of a point mass in pure gravity, which is given in [18] to all orders in $z(\omega)$. Here, we provide the first terms we need in this paper,

$$
\begin{aligned}
S_{\mathrm{int}}^{\mathrm{pm}}= & -\frac{m \kappa}{2} \int_{k} e^{i k \cdot b} \delta(k \cdot v) h_{\mu \nu}(-k) v^{\mu} v^{\nu}-i \frac{m \kappa}{2} \int_{k, \omega} e^{i k \cdot b} \delta(k \cdot v+\omega) h_{\mu \nu}(-k) z^{\rho}(-\omega)\left(2 \omega v^{(\mu} \delta_{\rho}^{\nu)}+v^{\mu} v^{\nu} k_{\rho}\right) \\
& -\frac{m \kappa^{2}}{4} \int_{k_{1}, k_{2}} e^{\left(k_{1}+k_{2}\right) \cdot b} \delta\left(\left(k_{1}+k_{2}\right) \cdot v\right) h_{\mu \rho}\left(-k_{1}\right) h_{\nu}^{\rho}\left(-k_{2}\right) v^{\mu} v^{\nu}+\mathcal{O}\left(k^{3}, z^{2}\right),
\end{aligned}
$$

from which we obtain the Feynman rules,

$$
k \downarrow \sum_{h_{\mu \nu}}=-i \frac{m \kappa}{2} e^{i k \cdot b} \delta(k \cdot v) v^{\mu} v^{\nu},
$$

$$
\begin{gathered}
k \downarrow \sum_{h_{\mu \nu}}^{\stackrel{\omega}{\longrightarrow}} z^{\rho}=\frac{m \kappa}{2} e^{i k \cdot b} \delta(k \cdot v+\omega) \\
\left(2 \omega v^{(\mu} \delta_{\rho}^{\nu)}+v^{\mu} v^{\nu} k_{\rho}\right),
\end{gathered}
$$




$$
\begin{aligned}
& h_{\mu \nu} \\
& h_{\rho \sigma} \\
& h_{1}
\end{aligned}=-\frac{m \kappa^{2}}{2} \int_{k_{1}, k_{2}} e^{i\left(k_{1}+k_{2}\right) \cdot b}
$$

where the parenthesis of Lorentz indices denotes symmetrization with unit weight, e.g., $v_{1}^{(\mu} v_{2}^{\nu)}=\frac{1}{2}\left(v_{1}^{\mu} v_{2}^{\nu}+v_{1}^{\nu} v_{2}^{\mu}\right)$.

\section{CLASSICAL DOUBLE COPY}

One of the main challenges of constructing the double copy in the classical limit of quantum field theories is that the locality structure is concealed. This is rooted in the classical limit of the massive scalar propagator [18], which contains both double and single propagators as we can see in WQFT from (14) and (15). Following [10], we tackle this difficulty by using the biadjoint scalar theory to identify the correct locality structure, i.e., disentangle the kinematical numerators from the propagator terms.

Another important strategy to establish the classical double copy is to consider more than two worldlines even if we are ultimately interested only in two-body interactions. This is to avoid the situation where some color factors in the two-body situation are vanishing, but the corresponding numerators do not, which under the double copy map may yield nonzero contributions. This may be evaded if we use as many worldlines as worldline-field interactions occur. Specifically, we consider an $(n+2)$ body system at $\mathrm{N}^{\mathrm{n}} \mathrm{LO}$. In the WQFT formalism, this is equivalent to taking into account only tree diagrams. To retrieve the binary system from this, we need to sum all possible ways of fusing the $(n+2)$ worldlines into two worldlines. In summary, our double copy relation of the eikonal phase at $\mathrm{N}^{(\mathrm{n}-1)} \mathrm{LO}$ reads

$$
\begin{aligned}
& \chi_{n}^{\mathrm{BS}}=-y^{2 n} \int \mathrm{d} \mu_{1,2, \ldots,(n+1)} \sum_{i, j} C_{i} K_{i j} \tilde{C}_{j}, \\
& \chi_{n}^{\mathrm{YM}}=-(i g)^{2 n} \int \mathrm{d} \mu_{1,2, \ldots,(n+1)} \sum_{i, j} C_{i} K_{i j} N_{j}, \\
& \chi_{n}^{\mathrm{DG}}=-\left(\frac{\kappa}{2}\right)^{2 n} \int \mathrm{d} \mu_{1,2, \ldots,(n+1)} \sum_{i, j} N_{i} K_{i j} N_{j},
\end{aligned}
$$

where $C_{i}, \tilde{C}_{j}$ denotes the color and dual-color factors, $N_{j}$ are the numerators, and $K_{i j}$ are the so-called double copy kernels that encode the locality structure. The sums extend over the dimensionalities of the numerators and the color factors. For further convenience, we have also defined the integral measure

$$
\mathrm{d} \mu_{1,2, \ldots, n}=\prod_{i=1}^{n}\left(\frac{\mathrm{d}^{4} k_{i}}{(2 \pi)^{4}} e^{i k_{i} \cdot b_{i}} \delta\left(k_{i} \cdot p_{i}\right)\right) \delta^{(4)}\left(\sum_{i=1}^{n} k_{i}^{\mu}\right),
$$

where $k_{i}$ is the total outgoing momentum of bosonic fields $\Phi(x)$ attached to a worldline. Note that we have defined the momentum of the massive particle as

$$
p_{i}^{\mu}:=m_{i} v_{i}^{\mu}, \quad \text { so that } \delta\left(k_{i} \cdot p_{i}\right)=\frac{\delta\left(k_{i} \cdot v_{i}\right)}{m_{i}} .
$$

Hereafter, we always express the numerator $N_{j}$ in terms of the momentum $p_{i}^{\mu}$ which is necessary in order to balance the mass dimension under the double copy. The kinematic numerators $N_{i}$ are arranged to satisfy the same algebraic equations as the color factors $C_{i}$,

$$
C_{i}+C_{j}+C_{k}=0 \Rightarrow N_{i}+N_{j}+N_{k}=0 .
$$

It is worth mentioning that we have the color-kinematic duality already at quartic order in the coupling constant.

\section{A. Eikonal at leading order (LO)}

The locality structure at leading order is trivial, so we do not need to employ the biadjoint scalar theory in order to double copy YM color charged particles to DG ones. In Yang-Mills coupled WQFT (WYM) the eikonal phase at this order involves only one diagram. Using the Feynman rules (17) and the gluon propagator (A4), we have

$$
i \chi_{1}^{\mathrm{YM}}=k_{1} \downarrow \xi^{1}=i g^{2} \int \mathrm{d} \mu_{1,2} \frac{\left(p_{1} \cdot p_{2}\right)\left(c_{1} \cdot c_{2}\right)}{k_{1}^{2}}
$$

where we have massaged the formula to fit the form as (30b). We can identify the color factor, the numerator, and the double copy kernel as

$$
C=\left(c_{1} \cdot c_{2}\right), \quad N=\left(p_{1} \cdot p_{2}\right), \quad K=\frac{1}{k_{1}^{2}} .
$$

In worldline coupled dilaton gravity (WDG), thanks to the decoupling of $\varphi$ from the worldline, we have also only one diagram mediated by $h_{\mu \nu}$. With (27) and the graviton propagator (A12), we obtain

$$
i \chi_{1}^{\mathrm{DG}}=\frac{1-k_{1} \downarrow}{2-\cdots}=\frac{-i \kappa^{2}}{4} \int \mathrm{d} \mu_{1,2} \frac{\left(p_{1} \cdot p_{2}\right)^{2}}{k_{1}^{2}} .
$$

Hence, at the leading order the eikonal of Yang-Mills and dilaton gravity obviously possess a double copy relation (30).

\section{B. Eikonal at next-to-lading order (NLO)}

As explained before, at next-to-leading order, to avoid the vanishing of some contributions in worldline coupled biadjoint scalar theory (WBS) and Yang-Mills coupled 
WQFT theory, we consider three worldlines. At this order, the locality structure is nontrivial. As we see, the double copy kernel is off diagonal. Therefore, we first consider the biadjoint scalar theory to identify the kernel. The Feynman diagrams in WBS can be calculated using the Feynman rules (22)-(25) and the three-point vertex of $\phi_{a \tilde{a}}$ (A9),

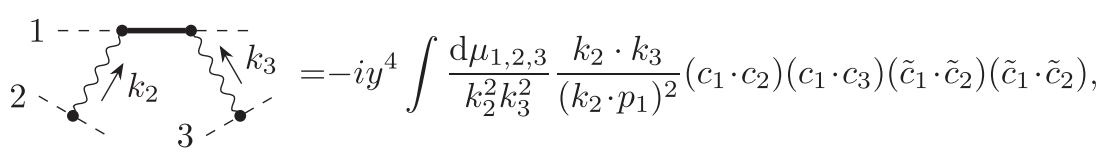

$$
2 、\left\{\begin{array}{l}
k_{2} \\
k_{3}
\end{array}\right\}^{k_{3}}=-i y^{4} \int \frac{\mathrm{d} \mu_{1,2,3}}{k_{2}^{2} k_{3}^{2}} \frac{1}{k_{2} \cdot p_{1}}\left(\left(c_{1}^{b a} c_{2}^{a} c_{3}^{b}\right)\left(\tilde{c}_{1} \cdot \tilde{c}_{2}\right)\left(\tilde{c}_{1} \cdot \tilde{c}_{3}\right)+\left(c_{1} \cdot c_{2}\right)\left(c_{1} \cdot c_{3}\right)\left(\tilde{c}_{1}^{\tilde{a}} \tilde{a} \tilde{c}_{2}^{\tilde{a}} \tilde{c}_{3}^{\tilde{b}}\right)\right) \text {, }
$$

$$
2
$$

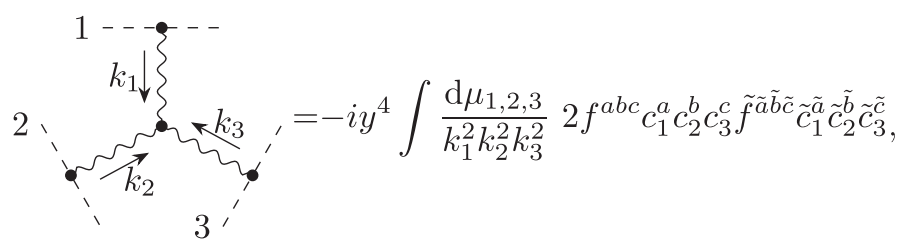

where for compactness we have defined

$$
c^{a b}:=\left(\psi^{\dagger} T^{a} T^{b} \psi\right), \quad \tilde{c}^{\tilde{a} \tilde{b}}:=\left(\tilde{\psi}^{\dagger} \tilde{T}^{\tilde{a}} \tilde{T}^{\tilde{b}} \tilde{\psi}\right) .
$$

Note that in (38) and (39), the propagator with an arrow denotes either the color or dual-color wave function, and we have added up their contributions. We stress that the factors $c^{a b}$ are absent in the equation of motion, so they will not explicitly appear in the classical solutions [29]. In fact, summing up (38) and (39), we can remove $c^{a b}$ by

$$
c^{a b}-c^{b a}=f^{a b c} c^{c},
$$

and similarly for the dual-color sector. However, these factors turn out to be critical for the double copy; because of them, we find classical numerators that satisfy colorkinematics duality at this order. From (37)-(39), we can identify three (dual-)color factors,

$$
C_{i}^{(123)}=\left\{\left(c_{1} \cdot c_{2}\right)\left(c_{1} \cdot c_{3}\right),\left(c_{1}^{a b} c_{2}^{a} c_{3}^{b}\right),\left(c_{1}^{b a} c_{2}^{a} c_{3}^{b}\right)\right\},
$$

$$
\tilde{C}_{i}^{(123)}=\left\{\left(\tilde{c}_{1} \cdot \tilde{c}_{2}\right)\left(\tilde{c}_{1} \cdot \tilde{c}_{3}\right),\left(\tilde{c}_{1}^{\tilde{a} \tilde{b}} \tilde{c}_{2}^{\tilde{a}} \tilde{c}_{3}^{\tilde{b}}\right),\left(\tilde{c}_{1}^{\tilde{b}} \tilde{a} \tilde{c}_{2}^{\tilde{a}} \tilde{c}_{3}^{\tilde{b}}\right)\right\}
$$

Note that here we only consider diagrams with worldline propagators of particle 1 . There are also contributions involving propagators of 2 and 3 , which can be gained simply by relabeling (123) in (37)-(39) and give us another six color factors. Together with the single (dual)-color factor emerging from (40)

$$
C_{i}^{(0)}=f^{a b c} c_{1}^{a} c_{2}^{b} c_{3}^{c}, \quad \tilde{C}_{i}^{(0)}=\tilde{f} \tilde{a} \tilde{a} \tilde{b} \tilde{c} \tilde{c} \tilde{1} \tilde{c}_{2}^{\tilde{b}} \tilde{c}_{3}^{\tilde{c}},
$$

we see that the double copy kernel $K_{i j}$ is 10 dimensional. Fortunately, $K_{i j}$ is block diagonal. The block that corresponds to the three-dimensional space (43) is

$$
K_{i j}^{(123)}=\frac{1}{k_{2}^{2} k_{3}^{2}}\left(\begin{array}{ccc}
\frac{k_{2} \cdot k_{3}}{\left(k_{2} \cdot p_{1}\right)^{2}} & \frac{-1}{k_{2} \cdot p_{1}} & \frac{1}{k_{2} \cdot p_{1}} \\
\frac{-1}{k_{2} \cdot p_{1}} & 0 & 0 \\
\frac{1}{k_{2} \cdot p_{1}} & 0 & 0
\end{array}\right),
$$


and analogously for the color dual (44). By permutations of (123), we may obtain other blocks. The last block coupling to the structure constant is extracted from (40) and is one dimensional; we have

$$
K_{i j}^{(0)}=\frac{2}{k_{1}^{2} k_{2}^{2} k_{3}^{2}} .
$$

We now proceed to consider the Yang-Mills coupled WQFT (WYM) theory. The Feynman diagrams are very similar to those of WBS theory. With the WYM Feynman rules (17)-(20), we may compute the contributions to the eikonal phase

$$
2
$$

$$
2
$$

$$
2
$$

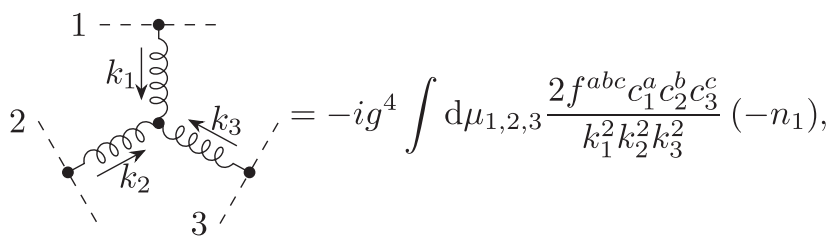

where we have defined

$$
\begin{gathered}
n_{0}=p_{1} \cdot p_{2} p_{1} \cdot p_{3}, \\
n_{1}=k_{2} \cdot p_{3} p_{1} \cdot p_{2}-k_{3} \cdot p_{2} p_{1} \cdot p_{3}-k_{2} \cdot p_{1} p_{2} \cdot p_{3} .
\end{gathered}
$$

Based on the color factors identified in (43), (45) and the double copy kernel (46), (47), we are led to organize the numerators as

$$
\begin{gathered}
N_{j}^{(123)}=\left\{n_{0}, \frac{-n_{1}}{2}, \frac{n_{1}}{2}\right\}, \\
N_{j}^{(0)}=-n_{1},
\end{gathered}
$$

so that the WYM eikonal may be decomposed in the form of (30b),

$$
\begin{aligned}
\chi_{2}= & -g^{4} \int \mathrm{d} \mu_{1,2,3} \sum_{i, j}\left(C_{i}^{(0)} K_{i j}^{(0)} N_{j}^{(0)}\right. \\
& \left.+\left(C_{i}^{(123)} K_{i j}^{(123)} N_{j}^{(123)}+\text { cyclic }\right)\right) .
\end{aligned}
$$

Fortunately, this decomposition automatically satisfies the color-kinematics duality

$$
\begin{gathered}
c_{1}^{a b} c_{2}^{a} c_{3}^{b}-c_{1}^{b a} c_{2}^{a} c_{3}^{b}=f^{a b c} c_{1}^{a} c_{2}^{b} c_{3}^{c} \\
\frac{-n_{1}}{2}-\frac{n_{1}}{2}=-n_{1} .
\end{gathered}
$$

Note that the decomposition of $N_{j}^{(123)}$ is not unique due to the Jacobi relation (42). ${ }^{2}$ We note that the color-kinematic duality is satisfied automatically up to this leading and next-to leading order if one uses Feynman gauge (this is not so in other gauges). We expect this property to break at higher orders in perturbation theory where the need of generalized gauge transformations arises. On then adds to the eikonal an arbitrary function multiplying the color factor Jacobi identity [Eq. (57)] in order to create a colorkinematic duality respecting representation.

In principle, we are now prepared to execute the double copy as proposed in (30c) to get the eikonal phase in the worldline coupled dilaton-gravity theory (WDG). In order to check the validity of our double copy prescription, we directly compute the eikonal in WDG theory with (27)(29); we find for the graphs not involving bulk graviton interactions,

\footnotetext{
${ }^{2}$ For example, we could also have $N_{j}^{(123)}=\left(\begin{array}{lll}n_{0} & 0 & n_{1}\end{array}\right)$. Color-kinematic duality still holds, and the double copy gives the correct gravitational result. We have chosen to write $N_{j}^{(123)}$ in a symmetric form.
} 


$$
2 、\left\{\begin{array}{l}
k_{3}=\frac{-i \kappa^{4}}{16} \int \frac{\mathrm{d} \mu_{1,2,3}}{k_{2}^{2} k_{3}^{2}\left(k_{2} \cdot p_{1}\right)^{2}}\left(\left(k_{2} \cdot k_{3}\right)\left(p_{1} \cdot p_{2}\right)^{2}\left(p_{1} \cdot p_{3}\right)^{2}-4\left(k_{2} \cdot p_{1}\right)^{2}\left(p_{1} \cdot p_{2}\right)\left(p_{1} \cdot p_{3}\right)\left(p_{2} \cdot p_{3}\right)\right. \\
\left.-2\left(k_{3} \cdot p_{2}\right)\left(k_{2} \cdot p_{1}\right)\left(p_{1} \cdot p_{2}\right)\left(p_{1} \cdot p_{3}\right)^{2}+2\left(k_{2} \cdot p_{3}\right)\left(k_{2} \cdot p_{1}\right)\left(p_{1} \cdot p_{3}\right)\left(p_{1} \cdot p_{2}\right)^{2}\right),
\end{array}\right.
$$

$$
2 \cdot \sqrt[2]{k_{2}} \sum^{-1} \cdot 3=\frac{-i \kappa^{4}}{16} \int \frac{\mathrm{d} \mu_{1,2,3}}{k_{2}^{2} k_{3}^{2}}\left(2\left(p_{1} \cdot p_{2}\right)\left(p_{1} \cdot p_{3}\right)\left(p_{2} \cdot p_{3}\right)\right)
$$

Summing up the two diagrams (59) and (60), we can check that the result can be written as

$$
\frac{-i \kappa^{4}}{16} \int \frac{\mathrm{d} \mu_{1,2,3}}{k_{2}^{2} k_{3}^{2}}\left(\frac{k_{2} \cdot k_{3} n_{0}^{2}}{\left(k_{2} \cdot p_{1}\right)^{2}}+\frac{2 n_{0} n_{1}}{k_{2} \cdot p_{1}}\right)=\frac{-i \kappa^{4}}{16} \int \mathrm{d} \mu_{1,2,3} \sum_{i, j} N_{i}^{(123)} K_{i j}^{(123)} N_{j}^{(123)} .
$$

In the last line, we have arranged the result to the form of (30c) with the double copy kernel $K_{i j}^{(123)}$ and the numerator $N_{i}^{(123)}$ defined in (46) and (54), respectively. Turning to the bulk graviton interaction graphs thanks to the field redefinition of $\left\{\varphi, h_{\mu \nu}\right\}$, the three-graviton vertex (A5) is directly proportional to the square of the three-gluon vertex, so we can easily compute the last diagram which is manifestly a double copy of the WYM one,

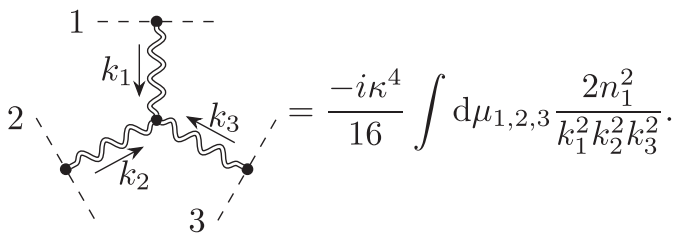

From (61) and (62), we therefore conclude that the double copy of the WYM eikonal coincides with the one of WDG also at the next-to-leading order $\left(\mathcal{O}\left(\kappa^{4}\right)\right)$.

\section{RADIATIVE DOUBLE COPY}

In this paper, we are mainly considering the conservative sector of the WQFT; however, with a slight modification we can generalize the eikonal double copy (30) to classical radiation. In WQFT, the $\Phi$ field radiation is computed as $[25,26]$

$$
-\left.i k^{2}\langle\Phi(k)\rangle\right|_{k^{2}=0}
$$

For $\Phi \in\left\{A_{\mu}^{a}, h_{\mu \nu}\right\}$, we also need to contract it with the polarizations $\left\{\epsilon^{\mu}, \epsilon^{\mu \nu}\right\}$, respectively. We take the gluon radiation as an example. Loosely speaking, the radiation at order $\mathcal{O}\left(g^{2 n-1}\right)$ can be obtained from the eikonal phase at $\mathcal{O}\left(g^{2 n}\right)$ by cutting off one worldline. Diagrammatically, the gluon radiation of a binary source at leading order can be gained from (48)-(51) by cutting the propagator $k_{3}$ and identifying $k_{3}$ with the momentum of the radiated gluon. The on-shell condition $k_{3} \cdot \epsilon=0$ plays the same role as the $\delta\left(k_{3} \cdot p_{3}\right)$ in the measure of the eikonal phase. This ensures that the gluon radiation can be decomposed into $C_{i} K_{i j} N_{j}$, with $C_{i}$ attained from (43) and (40) by striping off $c_{3}, N_{i}$ from (54) and (55) by replacing $p_{3}^{\mu}$ by $\epsilon^{\mu}$, and $K_{i j}$ being identical to (46). From the same approach, we can also get the gravitational radiation and decompose it as $N_{i} K_{i j} N_{j}$. Therefore, we conclude that the double copy construction works for radiation, too. We note that this is equivalent to the approach considered by Shen [10] and Goldberger and Ridgway [9] where the radiation is calculated by solving the equations of motion.

\section{FROM AMPLITUDE TO EIKONAL}

The expectation values in WQFT are directly linked to the classical limit of the S-matrix element. Consequently, we can expect that the classical double copy of WQFT discussed is also closely related to the double copy at the level of the scattering amplitude. In this section, we consider scalar quantum chromodynamics (SQCD), i.e., massive scalar fields coupled to Yang-Mills whose double copy has been studied in [30]. We claim that the classical limit of the scattering amplitude of $n$ distinct scalar pairs corresponds to the WYM eikonal phase at $\mathcal{O}\left(g^{2(n-1)}\right)$, and show the connection explicitly at $\mathcal{O}\left(g^{4}\right)$. Moreover, we demonstrate that the double copy of the eikonal phase is the classical limit of the BCJ double copy of the scattering amplitude.

The exponentiated eikonal phase is directly related to the classical limit of scattering amplitude [31,32],

$$
\left(1+\Delta_{q}\right) e^{i \chi}-1=\sum_{n=2} \frac{1}{2^{n}} \int \mathrm{d} \mu_{1,2, \ldots, n} \lim _{\hbar \rightarrow 0} \mathcal{A}(n \rightarrow n),
$$


where $\mathcal{A}(n \rightarrow n)$ denotes an amplitude of $n$ pairs of distinct massive scalars, and $\chi$ is the total eikonal phase, which scales as $\hbar^{-1}$ and receives contributions from all higherloop amplitudes. The introduction of the "quantum remainder" $\Delta_{q}$ (scaling as $\hbar^{n \geq 0}$ ) is needed for consistency [33]. Here, we only care about tree diagrams; therefore, we have

$$
\chi_{n-1}=\frac{-i}{2^{n}} \int \mathrm{d} \mu_{1,2, \ldots, n} \lim _{\hbar \rightarrow 0} \mathcal{A}^{\text {tree }}(n \rightarrow n) .
$$

The correspondence at the $2 \rightarrow 2$ level is rather trivial, so we focus on the $3 \rightarrow 3$ case. The leading order 6 -scalar amplitude in SQCD is $[30]^{3}$

$$
\begin{gathered}
\mathcal{A}^{\text {tree }}(3 \rightarrow 3)=\hat{p}_{2}+\frac{k_{2}}{2}, l \rightarrow \frac{k_{1}}{2}, j \\
\hat{p}_{3}+\frac{k_{3}}{2}, n \\
=\frac{8 \hat{c}^{(0)} \hat{n}^{(0)}}{k_{1}^{2} k_{2}^{2} k_{3}^{2}}+\left[\frac { 8 } { k _ { 2 } ^ { 2 } k _ { 3 } ^ { 2 } } \left(\frac{\hat{p}^{(123)} \hat{n}^{(123)}}{2 \hat{p}_{1} \cdot k_{2}-k_{2} \cdot k_{3}}\right.\right. \\
\left.\left.+\frac{\hat{c}^{(132)} \hat{n}^{(132)}}{2 \hat{p}_{1} \cdot k_{3}-k_{3} \cdot k_{2}}\right)+ \text { cyclic }\right],
\end{gathered}
$$

where we have introduced $\hat{p}_{i}$ as the average of the ingoing and outgoing momentum of particle $i$ which is orthogonal to its momentum transfer $\hat{p}_{i} \cdot k_{i}=0$. The color factors are

$$
\begin{gathered}
\hat{c}^{(0)}=f^{a b c} T_{i j}^{a} T_{k l}^{b} T_{m n}^{c}, \\
\hat{c}^{(123)}=\left(T^{b} T^{a}\right)_{i j} T_{k l}^{a} T_{m n}^{b}, \\
\hat{c}^{(132)}=\left(T^{a} T^{b}\right)_{i j} T_{k l}^{a} T_{m n}^{b},
\end{gathered}
$$

where $i, j, \ldots, l$ denote the color indices of the massive scalars. The corresponding numerators are

$$
\hat{n}^{(0)}=-i g^{4} \hat{p}_{1, \mu} \hat{p}_{2, \nu} \hat{p}_{3, \rho} V_{123}^{\mu \nu \rho},
$$

$$
\begin{aligned}
\hat{n}^{(123)}= & \frac{-i g^{4}}{2}\left(4 \hat{p}_{1} \cdot \hat{p}_{2} \hat{p}_{1} \cdot \hat{p}_{3}+2 \hat{p}_{1} \cdot \hat{p}_{3} k_{1} \cdot \hat{p}_{2}-2 \hat{p}_{1} \cdot \hat{p}_{2} k_{1} \cdot \hat{p}_{3}\right. \\
& \left.-2 \hat{p}_{1} \cdot k_{2} \hat{p}_{2} \cdot \hat{p}_{3}-k_{1} \cdot \hat{p}_{2} k_{1} \cdot \hat{p}_{3}+k_{2} \cdot k_{3} \hat{p}_{2} \cdot \hat{p}_{3}\right),
\end{aligned}
$$

$$
\begin{aligned}
\hat{n}^{(132)}= & \frac{-i g^{4}}{2}\left(4 \hat{p}_{1} \cdot \hat{p}_{2} \hat{p}_{1} \cdot \hat{p}_{3}+2 \hat{p}_{1} \cdot \hat{p}_{2} k_{1} \cdot \hat{p}_{3}-2 \hat{p}_{1} \cdot \hat{p}_{3} k_{1} \cdot \hat{p}_{2}\right. \\
& \left.-2 \hat{p}_{1} \cdot k_{3} \hat{p}_{2} \cdot \hat{p}_{3}-k_{1} \cdot \hat{p}_{2} k_{1} \cdot \hat{p}_{3}+k_{2} \cdot k_{3} \hat{p}_{2} \cdot \hat{p}_{3}\right),
\end{aligned}
$$

which has been brought into a form to satisfy colorkinematic duality $\hat{n}^{(132)}-\hat{n}^{(123)}=\hat{n}^{(0)}$. In the classical limit, we take small momentum transfers $k_{i} \rightarrow \hbar k_{i}$ and consider the expansion in small $\hbar$ following [19]. In (69)

\footnotetext{
${ }^{3} \mathrm{We}$ have converted the result of [30] to follow our conventions.
}

and (70), we have already sorted the terms in powers of $k_{i}$. We identify the momentum as $\hat{p}_{i}=p_{i}$, although since $\hat{p}_{i}^{2} \neq m_{i}^{2}$, we need to change the definition of $p_{i}$ to $p_{i}=$ $\hat{m}_{i} v_{i}$ with $\hat{m}_{i}^{2}=\hat{p}_{i}^{2}{ }^{4}$ At this order, the redefinition will not change the WQFT result. The massive propagators will become

$\frac{1}{2 \hat{p}_{1} \cdot k_{2}-k_{2} \cdot k_{3}} \rightarrow \frac{1}{\hbar} \frac{1}{2 p_{1} \cdot k_{2}}+\frac{k_{2} \cdot k_{3}}{4\left(p_{1} \cdot k_{2}\right)^{2}}+\mathcal{O}(\hbar)$.

Performing the classical limit of the Yang-Mills amplitude, we also need to consider the classical limit of the color factors, which was recently investigated by de la Cruz et al. [20]. Built on their insight, we propose the classical limit of the color factors to be

$$
\begin{gathered}
T_{i j}^{a} \rightarrow c^{a}, \\
\left(T^{a} T^{b}\right)_{i j} \rightarrow c^{a} c^{b}+\hbar c^{a b}, \\
f^{a b c} \rightarrow \hbar f^{a b c} .
\end{gathered}
$$

Note that the subleading term in (73) guarantees that the Jacobi identity holds in the classical limit.

It is now straightforward to compute the classical limit of the amplitude (66) and extract the eikonal using (65). Keeping only the leading order terms in the classical $\hbar \rightarrow 0$ limit, we have

$$
\frac{\hat{c}^{(0)} \hat{n}^{(0)}}{k_{1}^{2} k_{2}^{2} k_{3}^{2}} \rightarrow C_{i}^{(0)} K_{i j}^{(0)} N_{j}^{(0)}
$$

$$
\begin{aligned}
& \frac{1}{k_{2}^{2} k_{3}^{2}}\left(\frac{\hat{c}^{(123)} \hat{n}^{(123)}}{2 \hat{p}_{1} \cdot k_{2}-k_{2} \cdot k_{3}}+\frac{\hat{c}^{(132)} \hat{n}^{(132)}}{2 \hat{p}_{1} \cdot k_{3}-k_{2} \cdot k_{3}}\right) \\
& \quad \rightarrow C_{i}^{(123)} K_{i j}^{(123)} N_{j}^{(123)} .
\end{aligned}
$$

We therefore recover the eikonal phase of SQCD from the WQFT, which directly operates at the classical level.

We can double copy the SQCD amplitude (66) by replacing the color factors by the numerators. This results in an amplitude of massive scalars coupled to gravity and the dilaton. We can then likewise consider the classical limit of this gravitational amplitude,

$$
\frac{\hat{n}^{(0)} \hat{n}^{(0)}}{k_{1}^{2} k_{2}^{2} k_{3}^{2}} \rightarrow N_{i}^{(0)} K_{i j}^{(0)} N_{j}^{(0)}
$$

\footnotetext{
${ }^{4}$ This is related to the fact that we use Feynman propagators in the amplitudes, for the details, see [18].
} 


$$
\begin{aligned}
& \frac{1}{k_{2}^{2} k_{3}^{2}}\left(\frac{\hat{n}^{(123)} \hat{n}^{(123)}}{2 \hat{p}_{1} \cdot k_{2}-k_{2} \cdot k_{3}}+\frac{\hat{n}^{(132)} \hat{n}^{(132)}}{2 \hat{p}_{1} \cdot k_{3}-k_{2} \cdot k_{3}}\right) \\
& \quad \rightarrow N_{i}^{(123)} K_{i j}^{(123)} N_{j}^{(123)}
\end{aligned}
$$

which coincides with our calculation in WDG. We have therefore verified that the classical double copy of the worldline quantum field theory is in full agreement with the quantum double copy of amplitudes at LO and NLO. Note that the double copy of SQCD contains self-interactions of massive scalars [30]; however, these are short-range interactions and do not contribute to the classical theory. So we do not need to introduce additional terms in WDG, and the double copy automatically works out.

\section{CONCLUSIONS}

In this work, we have extended the WQFT formalism to massive, charged point particles coupled to the biadjoint scalar field, Yang-Mills, and dilaton-gravity theories. We proposed a classical double copy prescription for the eikonal phases, alias free energies, in these theories and explicitly verified the validity of the double copy up to quartic order (NLO) in the coupling constants. This entails the double copy relation for the particle's deflection (or scattering angle) as the derivative of the eikonal with respect to the impact parameter. With minor modifications, our double copy prescription also applies to classical radiation emitted in the scattering process. As a technical tool, it was necessary to increase the number of worldlines of scattered particles with the order of perturbation theory, i.e., an $(n+2)$-body system for the $\mathrm{N}^{n} \mathrm{LO}$ order as well as to consider the eikonal of WBS in parallel to establish the double copy kernels. In fact, we expect all expectation values in WYM and WDG to feature the double copy relation as they are directly related to the quantum scattering amplitudes. To illustrate the connection, we compared our eikonal phase to the classical limit of the corresponding eikonal emerging from the massive scalar six-point amplitude finding agreement.

These insights give us the expectation that the classical double copy for the WQFT will prevail to NNLO and higher. This would cure the breakdown observed in [17] of a double copy prescription for the off-shell effective action of the particle's worldline coordinates $x^{\mu}(\tau)$. The essential difference of our approach to the off-shell effective action of $[16,17]$ is that in WQFT both the force mediating fields and the fluctuations on the worldline are integrated out in the path integral.

The most important application of WQFT will be to Einstein gravity. This, however, continues to be a challenge for the double copy prescription as it suffers from pollution of the dilaton and, in principle, even the Kalb-Ramond twoform. Many approaches have been explored to remove the dilaton from the double copy construction [21,34-36]. Since the WQFT provides a simple way to extract classical quantities, it might be easier to project out dilaton at the classical level. It will be interesting to explore this in future work.

An obvious extension of the classical double copy is to incorporate spin, and some attempts in this direction are $[12,13,37]$. The recently discovered hidden supersymmetry in the worldline description of spinning compact bodies [24] should be applicable to the Yang-Mills case as well. In particular the limitation of coupling higher-spin worldline theories to gravity might be overcome upon using the double copy. Concretely, the construction of the $\sqrt{\text { Kerr }}$ solution [8] in the language of the WQFT would be an interesting starting point.

\section{ACKNOWLEDGMENTS}

This project has received funding from the European Union's Horizon 2020 research and innovation program under the Marie Sklodowska-Curie Grant Agreement No. 764850 "SAGEX". J.P. thanks the Max-PlanckInstitut für Physik (Werner-Heisenberg Institut) for hospitality. Some of our figures were produced with the help of TikZ-Feynman [38].

\section{APPENDIX A: CONVENTIONS}

We use the mostly minus signature for the spacetime metric. The generators and structure constants of the color gauge group are normalized such that

$$
\begin{gathered}
{\left[T^{a}, T^{b}\right]=f^{a b c} T^{c},} \\
\operatorname{Tr}\left(T^{a} T^{b}\right)=\frac{\delta^{a b}}{2} .
\end{gathered}
$$

Note that our $f^{a b c}$ is different from a more usual convention by a factor of $i$. For the YM theory, we use the standard Lagrangian $S^{\mathrm{YM}}=-\int \mathrm{d}^{4} x\left(F_{\mu \nu}^{a}\right)^{2} / 4$, where

$$
F_{\mu \nu}^{a}=\partial_{\mu} A_{\nu}^{a}-\partial_{\nu} A_{\mu}^{a}-i g f^{a b c} A_{\mu}^{b} A_{\nu}^{c} .
$$

In the Feynman gauge, the gluon propagator and the threegluon vertex are

$$
A_{\mu}^{a} \stackrel{k}{\stackrel{k}{\longrightarrow}} A_{\nu}^{b}=\frac{-i}{k^{2}} \eta_{\mu \nu} \delta^{a b}
$$

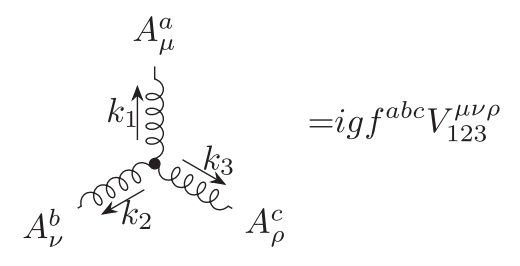


where

$V_{123}^{\mu \nu \rho}=\left[\eta^{\mu \nu}\left(k_{1}-k_{2}\right)^{\rho}+\eta^{\nu \rho}\left(k_{2}-k_{3}\right)^{\mu}+\eta^{\rho \mu}\left(k_{3}-k_{1}\right)^{\nu}\right]$.

The action of biadjoint scalar theory is

$S^{\mathrm{BS}}=\int \mathrm{d}^{4} x\left(\frac{1}{2}\left(\partial_{\mu} \phi^{a \tilde{a}}\right)^{2}-\frac{y}{3} f^{a b c} \tilde{f}^{\tilde{a} \tilde{b} \tilde{c}} \phi_{a \tilde{a}} \phi_{b \tilde{b}} \phi_{c \tilde{c}}\right)$.

The Feynman rules are

$$
\phi^{a \tilde{a}} \stackrel{k}{\sim} \phi^{b \tilde{b}}=\frac{i}{k^{2}},
$$

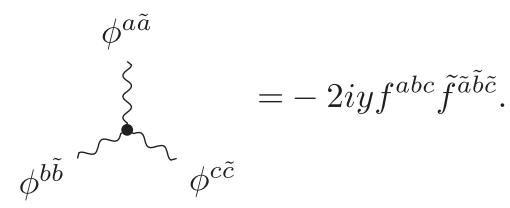

For dilaton gravity, we strictly follow the convention in [16] which extensively simplifies our calculation. Originally, the action is

$$
S^{\mathrm{DG}}=-\frac{2}{\kappa^{2}} \int \mathrm{d}^{4} x \sqrt{-g}\left[R-2 \partial_{\mu} \varphi \partial^{\mu} \varphi\right] .
$$

We expand it in the weak field limit (4). Using the field redefinition of $\left\{\varphi, h_{\mu \nu}\right\}$ and the gauge defined in [16], we rewrite the action as

$$
\begin{aligned}
S^{\mathrm{DG}}= & \int \mathrm{d}^{4} x\left(\frac{1}{2} \partial_{\rho} h_{\mu \nu} \partial^{\rho} h^{\mu \nu}\right. \\
& \left.+\frac{\kappa}{4 \cdot 3 !} \mathcal{V}_{123}^{\mu \alpha \gamma} \mathcal{V}_{123}^{\nu \beta \delta} h_{1 \mu \nu} h_{2 \alpha \beta} h_{3 \gamma \delta}+\mathcal{O}\left(\kappa^{2}, \varphi\right)\right),
\end{aligned}
$$

where $\mathcal{V}_{123}^{\nu \beta \delta}=\left.V_{123}^{\nu \beta \delta}\right|_{k_{i} \rightarrow \partial_{i}}$ is the position space version of $V_{123}^{\nu \beta \delta}$, with the labels $1,2,3$ indicating on which $h_{\mu \nu}$ the partial derivatives should be applied. This yields the graviton propagator

$$
h_{\mu \nu} \stackrel{k}{\stackrel{k u e}{\longrightarrow}} h_{\rho \sigma}=\frac{i}{k^{2}} P_{\mu \nu \rho \sigma}
$$

with

$$
P_{\mu \nu \rho \sigma}=\frac{\eta_{\mu \rho} \eta_{\nu \sigma}+\eta_{\mu \sigma} \eta_{\nu \rho}}{2},
$$

and the three-graviton vertex is simply

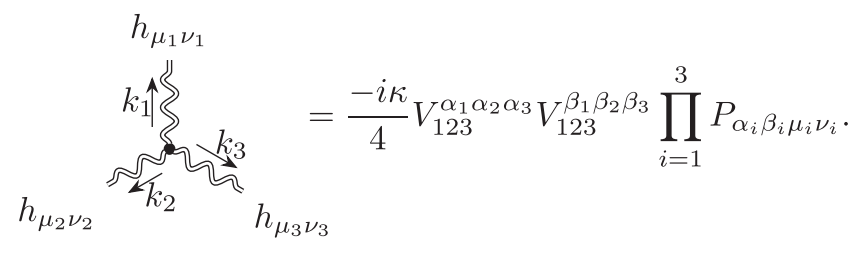

From (A5) and (A14), we can already see the double copy structure of the vertices.

\section{APPENDIX B: EIKONAL AND MOMENTUM DEFLECTION}

Here, we provide a simple proof in the path integral formalism that the partition function $\mathcal{Z}_{\mathrm{WQFT}}$ can be identified with the exponentiated eikonal phase. Let us first consider the derivative of $\ln \mathcal{Z}_{\mathrm{WQFT}}$ with respect to $b_{i}^{\mu}$,

$i \frac{\partial \ln \mathcal{Z}_{\mathrm{WQFT}}}{\partial b_{i}^{\mu}}=\left\langle-\frac{\partial S^{\mathrm{WQFT}}}{\partial b_{i}^{\mu}}\right\rangle=-\int_{-\infty}^{+\infty} \mathrm{d} \tau\left\langle\frac{\partial L^{\mathrm{pp}}}{\partial x_{i}^{\mu}}\right\rangle$,

where in the last step we exploit the fact that in the full action $b_{i}^{\mu}$ only appears as the $\tau$-independent background of $x_{i}^{\mu}(\tau)$ in the point particle action $S^{\mathrm{pp}}=\int \mathrm{d} \tau L^{\mathrm{pp}}$, where $L^{\mathrm{pp}}$ is the Lagrangian. As the expectation value of the equation of motion for $x(\tau)$ vanishes, we can rewrite Eq. (B1) as

$$
i \frac{\partial \ln \mathcal{Z}_{\mathrm{WQFT}}}{\partial b_{i}^{\mu}}=-\int_{-\infty}^{+\infty} \mathrm{d} \tau\left\langle\frac{\mathrm{d}}{\mathrm{d} \tau} \frac{\partial L^{\mathrm{pp}}}{\partial \dot{x}_{i}^{\mu}}\right\rangle=\left.\left\langle p_{i, \mu}^{\mathrm{can}}\right\rangle\right|_{-\infty} ^{+\infty},
$$

where $p_{i, \mu}^{\mathrm{can}}=-\partial L^{\mathrm{pp}} / \partial \dot{x}_{i}^{\mu}$ is the canonical momentum conjugated to $x^{\mu}$. Since we are studying a scattering process, in past and future infinity we may assume that the point particles are so far separated that the interaction terms vanish. In this case, $p_{i, \mu}^{\text {can }}$ reduces to the kinematic momentum $m_{i} \dot{x}_{i}^{\mu}$, so we have

$$
m_{i} \Delta \dot{x}_{i}^{\mu}=i \frac{\partial \ln \mathcal{Z}_{\mathrm{WQFT}}}{\partial b_{i, \mu}}
$$

Therefore in this paper, we define the generalized eikonal phase for more than two worldlines as

$$
\chi=-i \ln \mathcal{Z}_{\mathrm{WQFT}} .
$$


[1] Z. Bern, J. J. M. Carrasco, and H. Johansson, Phys. Rev. D 78, 085011 (2008).

[2] Z. Bern, J. J. M. Carrasco, and H. Johansson, Phys. Rev. Lett. 105, 061602 (2010).

[3] Z. Bern, J. J. Carrasco, M. Chiodaroli, H. Johansson, and R. Roiban, arXiv:1909.01358.

[4] N. E. J. Bjerrum-Bohr, P. H. Damgaard, T. Sondergaard, and P. Vanhove, J. High Energy Phys. 01 (2011) 001; C. R. Mafra, O. Schlotterer, and S. Stieberger, J. High Energy Phys. 07 (2011) 092; N. E. J. Bjerrum-Bohr, J. L. Bourjaily, P. H. Damgaard, and B. Feng, J. High Energy Phys. 09 (2016) 094; Y.-J. Du and F. Teng, J. High Energy Phys. 04 (2017) 033; L. de la Cruz, A. Kniss, and S. Weinzierl, J. High Energy Phys. 03 (2018) 064; E. Bridges and C. R. Mafra, J. High Energy Phys. 10 (2019) 022; M. Reiterer, arXiv:1912.03110.

[5] R. Monteiro and D. O'Connell, J. High Energy Phys. 07 (2011) 007; C. Cheung and C.-H. Shen, Phys. Rev. Lett. 118, 121601 (2017); G. Chen, H. Johansson, F. Teng, and T. Wang, J. High Energy Phys. 11 (2019) 055; 10 (2021) 042.

[6] L. J. Dixon, in Theoretical Advanced Study Institute in Elementary Particle Physics (TASI 95): QCD and Beyond (1996), pp. 539-584; H. Elvang and Y.-t. Huang, arXiv:1308.1697; J. M. Henn and J. C. Plefka, Scattering Amplitudes in Gauge Theories (Springer, Berlin, 2014), Vol. 883.

[7] R. Monteiro, D. O'Connell, and C. D. White, J. High Energy Phys. 12 (2014) 056; A. Luna, R. Monteiro, D. O'Connell, and C. D. White, Phys. Lett. B 750, 272 (2015); A. Luna, R. Monteiro, I. Nicholson, A. Ochirov, D. O'Connell, N. Westerberg, and C. D. White, J. High Energy Phys. 04 (2017) 069; M. Carrillo-González, R. Penco, and M. Trodden, J. High Energy Phys. 04 (2018) 028; A. Guevara, B. Maybee, A. Ochirov, D. O'connell, and J. Vines, J. High Energy Phys. 03 (2021) 201; R. Monteiro, D. O'Connell, D. P. Veiga, and M. Sergola, J. High Energy Phys. 05 (2021) 268; C. D. White, Phys. Rev. Lett. 126, 061602 (2021); E. Chacón, S. Nagy, and C. D. White, J. High Energy Phys. 05 (2021) 239; H. Godazgar, M. Godazgar, R. Monteiro, D. P. Veiga, and C. N. Pope, arXiv: 2109.07866.

[8] N. Arkani-Hamed, Y.-t. Huang, and D. O'Connell, J. High Energy Phys. 01 (2020) 046.

[9] W. D. Goldberger and A. K. Ridgway, Phys. Rev. D 95, 125010 (2017).

[10] C.-H. Shen, J. High Energy Phys. 11 (2018) 162.

[11] W. D. Goldberger and A. K. Ridgway, Phys. Rev. D 97, 085019 (2018).

[12] W. D. Goldberger, J. Li, and S. G. Prabhu, Phys. Rev. D 97, 105018 (2018).

[13] W. D. Goldberger and J. Li, J. High Energy Phys. 02 (2020) 092.

[14] W. D. Goldberger, S. G. Prabhu, and J. O. Thompson, Phys. Rev. D 96, 065009 (2017).

[15] F. Bastianelli, F. Comberiati, and L. de la Cruz, arXiv: 2107.10130.

[16] J. Plefka, J. Steinhoff, and W. Wormsbecher, Phys. Rev. D 99, 024021 (2019).
[17] J. Plefka, C. Shi, J. Steinhoff, and T. Wang, Phys. Rev. D 100, 086006 (2019).

[18] G. Mogull, J. Plefka, and J. Steinhoff, J. High Energy Phys. 02 (2021) 048.

[19] D. A. Kosower, B. Maybee, and D. O'Connell, J. High Energy Phys. 02 (2019) 137.

[20] L. de la Cruz, B. Maybee, D. O'Connell, and A. Ross, J. High Energy Phys. 12 (2020) 076.

[21] Z. Bern, C. Cheung, R. Roiban, C.-H. Shen, M. P. Solon, and M. Zeng, J. High Energy Phys. 10 (2019) 206.

[22] T. Damour, Phys. Rev. D 97, 044038 (2018); P. Di Vecchia, C. Heissenberg, R. Russo, and G. Veneziano, J. High Energy Phys. 07 (2021) 169; N. E. J. Bjerrum-Bohr, P. H. Damgaard, G. Festuccia, L. Planté, and P. Vanhove, Phys. Rev. Lett. 121, 171601 (2018); C. Cheung, I. Z. Rothstein, and M. P. Solon, Phys. Rev. Lett. 121, 251101 (2018); Z. Bern, C. Cheung, R. Roiban, C.-H. Shen, M. P. Solon, and M. Zeng, Phys. Rev. Lett. 122, 201603 (2019); N. E. J. Bjerrum-Bohr, P. H. Damgaard, L. Planté, and P. Vanhove, arXiv:2105.05218; E. Herrmann, J. Parra-Martinez, M. S. Ruf, and M. Zeng, arXiv:2104.03957; A. Brandhuber, G. Chen, G. Travaglini, and C. Wen, arXiv:2108.04216; Z. Bern, J. Parra-Martinez, R. Roiban, M. S. Ruf, C.-H. Shen, M. P. Solon, and M. Zeng, Phys. Rev. Lett. 126, 171601 (2021); A. Cristofoli, R. Gonzo, D. A. Kosower, and D. O’Connell, arXiv:2107.10193.

[23] R. A. Porto, Phys. Rep. 633, 1 (2016); M. Levi, Rep. Prog. Phys. 83, 075901 (2020); G. Kälin and R. A. Porto, J. High Energy Phys. 11 (2020) 106; G. Kälin, Z. Liu, and R. A. Porto, Phys. Rev. Lett. 125, 261103 (2020); C. Dlapa, G. Kälin, Z. Liu, and R. A. Porto, arXiv:2106.08276.

[24] G. U. Jakobsen, G. Mogull, J. Plefka, and J. Steinhoff, arXiv:2109.04465.

[25] G. U. Jakobsen, G. Mogull, J. Plefka, and J. Steinhoff, Phys. Rev. Lett. 126, 201103 (2021).

[26] G. U. Jakobsen, G. Mogull, J. Plefka, and J. Steinhoff, arXiv: 2106.10256.

[27] F. Bastianelli, R. Bonezzi, O. Corradini, and E. Latini, J. High Energy Phys. 10 (2013) 098.

[28] F. Bastianelli, R. Bonezzi, O. Corradini, E. Latini, and K. H. Ould-Lahoucine, J. Phys. Conf. Ser. 1208, 012004 (2019).

[29] S. K. Wong, Nuovo Cimento A 65, 689 (1970).

[30] J. Plefka, C. Shi, and T. Wang, Phys. Rev. D 101, 066004 (2020).

[31] D. Amati, M. Ciafaloni, and G. Veneziano, Phys. Lett. B 197, 81 (1987).

[32] D. Amati, M. Ciafaloni, and G. Veneziano, Nucl. Phys. B347, 550 (1990).

[33] C. Heissenberg, Phys. Rev. D 104, 046016 (2021).

[34] A. Luna, I. Nicholson, D. O'Connell, and C. D. White, J. High Energy Phys. 03 (2018) 044.

[35] H. Johansson and A. Ochirov, J. High Energy Phys. 11 (2015) 046.

[36] J. J. M. Carrasco and I. A. Vazquez-Holm, J. High Energy Phys. 11 (2021) 088.

[37] J. Li and S. G. Prabhu, Phys. Rev. D 97, 105019 (2018).

[38] J. Ellis, Comput. Phys. Commun. 210, 103 (2017). 